\title{
Conteo de marcas de yemas para estimar la edad en Polylepis tarapacana: Una técnica potencial
}

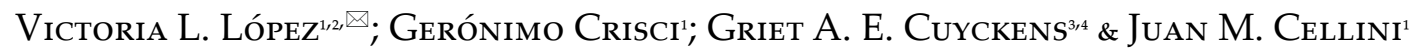 \\ ${ }^{1}$ Laboratorio de Investigaciones en Maderas, Facultad de Ciencias Agrarias y Forestales, Universidad Nacional de La Plata. \\ ${ }^{2}$ CCT La Plata (CONICET-La Plata). ${ }^{3}$ Instituto de Ecorregiones Andinas, Universidad Nacional de Jujuy (UNJU). ${ }^{4}$ Centro de \\ Estudios Territoriales Ambientales y Sociales (UNJU).
}

\begin{abstract}
Resumen. En las especies forestales que presentan un crecimiento determinado, las yemas generan marcas que se usan para estimar las edades a lo largo de brotes apicales o de ramas, mediante el conteo de entrenudos. Para estimar la edad a lo largo de las ramas en especies amenazadas y de crecimiento lento, como es el caso de Polylepis tarapacana Phil., es crucial contar con técnicas no destructivas que eviten colectar ramas, renovales o ejemplares completos. Por lo tanto, el objetivo de este trabajo fue evaluar la potencialidad de la técnica de estimación de la edad que utiliza las marcas externas a lo largo de la rama en $P$. tarapacana. Se trabajó con 13 ramas apicales colectadas en 13 bosques de la localidad de Lagunillas del Farallón, Dto. de Rinconada. En cada una de las ramas se realizó el conteo de marcas de brotes utilizando dos métodos: a) a lo largo de los 20 $\mathrm{cm}$ de longitud de la rama principal y b) en un brote lateral de la rama principal, se contaron tres marcas a lo largo del brote. Se analizó si el conteo de marcas se ajusta a la edad con un modelo linear generalizado mixto $(\mathrm{Glmm})$, incluyendo el factor aleatorio árbol $(n=26)$. Los resultados indican que existe potencial para aplicar esta técnica, pero para generar un modelo confiable sería necesario colectar un mayor número de muestras en futuras investigaciones.
\end{abstract}

[Palabras clave: cicatrices de yemas, Jujuy, Altos Andes, crecimiento determinado]

\begin{abstract}
Bud mark count to estimate age in Polylepis tarapacana: A potential technique. In forest species that present a determined growth, the buds generate marks that are used to estimate the ages along the apical shoots or branches, by counting the internodes. In order to estimate the age along the branches in threatened and slow-growing species, such as Polylepis tarapacana Phil., it is crucial to have non-destructive techniques that avoid the collection of branches, sapling or complete specimens. Therefore, the objective of this work was to evaluate the potentiality of the age estimation technique, which uses external marks along the branch in $P$. tarapacana. We worked with 13 apical branches collected in 13 forests in the town of Lagunillas del Farallón, Dpt. Rinconada. Shoot marks were counted on each branch using two methods: a) along the $20 \mathrm{~cm}$ length of the main branch and b) on a lateral shoot of the main branch, three marks were counted along the length of the shoot. It was analyzed if the mark count adjusts to age with a mixed generalized linear model (Glmm), including tree as a random factor $(n=26)$. The results indicate that there is potential for applying this technique, but in order to generate a reliable model, it would be necessary to collect a larger number of samples in future research.
\end{abstract}

\section{INTRODUCCIÓN}

El crecimiento en altura en árboles y arbustos resulta de la expansión de brotes terminales en el tallo principal y sus ramas, los cuales suelen experimentar un crecimiento determinado ('fixed growth' sensu Kozlowski and Pallardy [1997] o 'determinated growth' sensu Hallé et al. [1978]), donde estos brotes crecen luego de un período de descanso, generando marcas externas dejadas por las yemas en ramas (Kozlowski and Pallardy 1997). Esta información se usa para estimar las edades a lo largo de brotes apicales o ramas, mediante el conteo de entrenudos (Kajimoto et al.1998; Hoch and Körner 2005) o de las marcas de yemas (Kajimoto et al. 1998; Cuevas 2002; Gea et al. 2004; Martínez Pastur et al. 2007; Soler et

Editora asociada: Ana Cingolani 凶victoria.lopez@agro.unlp.edu.ar al. 2018). A través de la combinación de la edad estimada y el largo de las ramas es posible calcular el crecimiento anual del individuo o de la rama evaluada en especies forestales que presenten un crecimiento determinado (Kajimoto et al.1998; Cuevas 2002; Gea et al. 2004; Hoch and Körner 2005; Martínez Pastur et al. 2007; Soler et al. 2018). Esta estimación se puede realizar sólo en ramas jóvenes, ya que el método suele volverse menos preciso por la formación de corteza que oculta las cicatrices en ramas más viejas (Kajimoto et al. 1998; Cuevas 2002). La precisión de las edades determinadas por este método no destructivo debe ser verificada contando los anillos anuales en cada base del tallo por medio de corte, pulido y observación en lupa (Kajimoto et al. 1998). 
El límite altitudinal superior de los bosques se encuentra bajo condiciones similares de temperatura a nivel mundial, pero los mecanismos funcionales que reducen el crecimiento de los árboles son poco conocidos (Toivonen et al. 2014). El crecimiento de los bosques en su límite altitudinal se encuentra determinado por factores climáticos, y a medida que aumenta la altitud, su estructura se modifica de árboles a arbustos dispersos (Hoch and Körner 2005); su mayor altitud ocurre en regiones secas como el Tibet (Miehe et al. 2003) y, en especial, en el altiplano sudamericano (Braun 1997).

El altiplano argentino está caracterizado por paisajes heterogéneos que poseen pastos bajos y matorrales con ocasionales bosquecillos monoespecíficos de Polylepis tarapacana Phil. (Kessler 1995). Esta especie es considerada casi amenazada (Near Threatened [NT]) (UICN 2020), debido a que las comunidades locales la usan como combustible (Renison et al. 2010). Las diferentes condiciones adversas presentes en el altiplano (e.g., alta radiación solar, evapotranspiración, elevada amplitud térmica, entre otras) son superadas optimizando el uso de sus recursos mediante modificaciones fisiológicas (ritmo de crecimiento, desarrollo) y morfológicas, tanto internas como externas (Cozzi and Moschione 2011). Además, la baja temperatura influye en el crecimiento (Hoch and Körner 2005), lo que resulta en una disminución de la densidad, el área basal y el tamaño de los árboles a medida que aumenta la altitud (Zhang et al. 2010).

Existen trabajos de crecimiento para Nothofagus pumilio (Poepp. and Endl.) Krasser y Nothofagus antarctica (G. Forst.) Ørsted en los que se calcula la edad a través de las marcas que dejan las yemas en el tallo de las plántulas, teniendo gran precisión hasta los 15-20 años de edad (Cuevas 2002). Asimismo, esta técnica fue utilizada en renovales de Pinus pumila (Pall.) Regel, en los que la edad de las plántulas se determinó contando el número de nodos correspondientes al crecimiento anual a lo largo de un tallo principal desde la porción basal hasta el brote terminal (Kajimoto et al. 1998). Hoch and Körner (2005) usaron una técnica similar en arboles de Polylepis tarapacana Phil., en los que, mediante el conteo de marcas de yemas y la longitud entre estas marcas en brotes de árboles, determinaron el crecimiento apical en dos períodos consecutivos, y utilizaron esta información para estudiar el crecimiento en un gradiente altitudinal en Sajama, Bolivia.
Sin embargo, estos autores no corroboraron la precisión de esta técnica relacionando la edad real y las marcas.

Para estimar la edad a lo largo de las ramas en especies amenazadas y de crecimiento lento, como es el caso de $P$. tarapacana, es crucial contar con técnicas no destructivas que eviten la colecta de ramas, renovales o ejemplares completos. A su vez, debido a las condiciones ambientales de los Altos Andes y su difícil acceso, es clave contar con una técnica que permita determinar la edad, a fin de optimizar el esfuerzo y tiempo. Por lo tanto, el objetivo de este trabajo fue evaluar la potencialidad de la técnica de estimación de la edad, que utiliza las marcas externas a lo largo de la rama, en $P$. tarapacana como caso de estudio.

\section{Materiales y Métodos}

\section{Sitio de muestreo}

Se trabajó en la localidad de Lagunillas del Farallón, Dto. de Rinconada, Provincia de Jujuy $\left(22^{\circ} 27^{\prime} 42^{\prime \prime} \mathrm{S}-66^{\circ} 37^{\prime} 40^{\prime \prime} \mathrm{O}\right)$. Esta localidad se encuentra en el extremo noroeste de la Provincia de Jujuy, a 4165 m s. n. m., en la Cuenca del Río Grande de San Juan y en el complejo endorreico de Lagunas de Vilama, correspondiendo el ambiente de estepas puneñas y alto andina (Wawrzyk and Vilá 2013). La zona de estudio presenta una temperatura media anual menor a 10 ${ }^{\circ} \mathrm{C}$, con una amplitud térmica diaria de 25 ${ }^{\circ} \mathrm{C}$. Las precipitaciones se concentran en los meses de verano (enero-febrero), y llegan a $200 \mathrm{~mm}$ de precipitación media anual (Mina Pirquitas 2004).

\section{Especie en estudio}

Dentro del género Polylepis, la especie $P$. tarapacana se desarrolla en la provincia fitogeográfica Altoandina. Forma parches poco densos y dispersos, en el rango altitudinal de 4400 a 5000 m s. n. m. (Kessler 2006; Renison et al. 2013), y crece en regiones con 100 a $500 \mathrm{~mm}$ de precipitación media anual, en exposiciones norte y noroeste (Renison et al. 2010; Mendoza and González 2011; Morales et al. 2012; Cuyckens et al. 2016). Puede crecer en todas las posiciones topográficas, mayormente en posiciones de ladera media a alta (Piña 2015). P. tarapacana es un árbol de hábito simpódico que presenta un tronco corto y puede alcanzar los $3 \mathrm{~m}$ de altura (Morales et al. 2004). Con respecto a su crecimiento 
en altura, el período de mayor elongación de sus brotes ocurre luego de la época de lluvia (marzo y abril), cuando la reducción de temperatura genera lluvias y nubosidad (Hoch and Körner 2005), lo que marca una estacionalidad en el crecimiento.

\section{Relevamiento a campo}

Mediante imágenes satelitales Bing (Microsoft, USA) de alta resolución se seleccionaron 13 formaciones forestales de $P$. tarapacana de la Provincia de Jujuy, que se desarrollan en un gradiente de exposición, de altitud, pendiente y cobertura forestal. Con el fin de caracterizar el hábitat donde se desarrolla esta especie se realizaron mediciones de parámetros en forma cuantitativa de variables ambientales, topográficas y de estructura forestal. Se instaló para cada bosque una parcela circular de radio variable, ubicando un punto al azar previo al muestreo y utilizando un dispositivo de geoposicionamiento global (GPS) en terreno. El radio de la parcela se ajustó a un total mínimo de 20 individuos de árboles y arbustos de $P$. tarapacana. Se midió el DAP máximo (DAP-cm a $1.3 \mathrm{~m}$ de altura del árbol de mayor tamaño) y la altura media dominante del bosque como promedio de los 3 árboles más altos (Assmann 1970). Se asignó un valor de cero al DAP máximo cuando ningún individuo de la parcela superaba el
$1.3 \mathrm{~m}$ de altura. Dentro de cada parcela se recolectó una rama apical de $20 \mathrm{~cm}$ de largo del individuo más cercano al centro de la parcela que presentase buena sanidad y cuya altura no fuera inferior a la altura media dominante. Cada rama seleccionada se almacenó en un sobre de papel rotulado con el fin de preservar y aislar la muestra.

En cada una de las 13 parcelas se caracterizó la pendiente, exposición y altitud con clinómetro, brújula y GPS. La exposición se analizó en el gradiente N-S transformando el valor de exposición mediante la función coseno, mientras que para la exposición EO se utilizó la función seno. Las parcelas muestreadas presentaron un amplio gradiente de altitud, en donde la especie se distribuye entre los 4304 y 4842 m s. n. m. Se calcularon los valores promedio de las variables topográficas y de estructura forestal de los 13 bosques de $P$. tarapacana estudiados. Los valores máximos y mínimos reflejan los valores extremos calculados como promedio dentro de cada parcela.

\section{Procesamiento de muestras}

El material recolectado de $P$. tarapacana se analizó en laboratorio. En cada una de las 13 ramas se realizó el conteo de marcas de brotes utilizando dos métodos: a) a lo largo de los

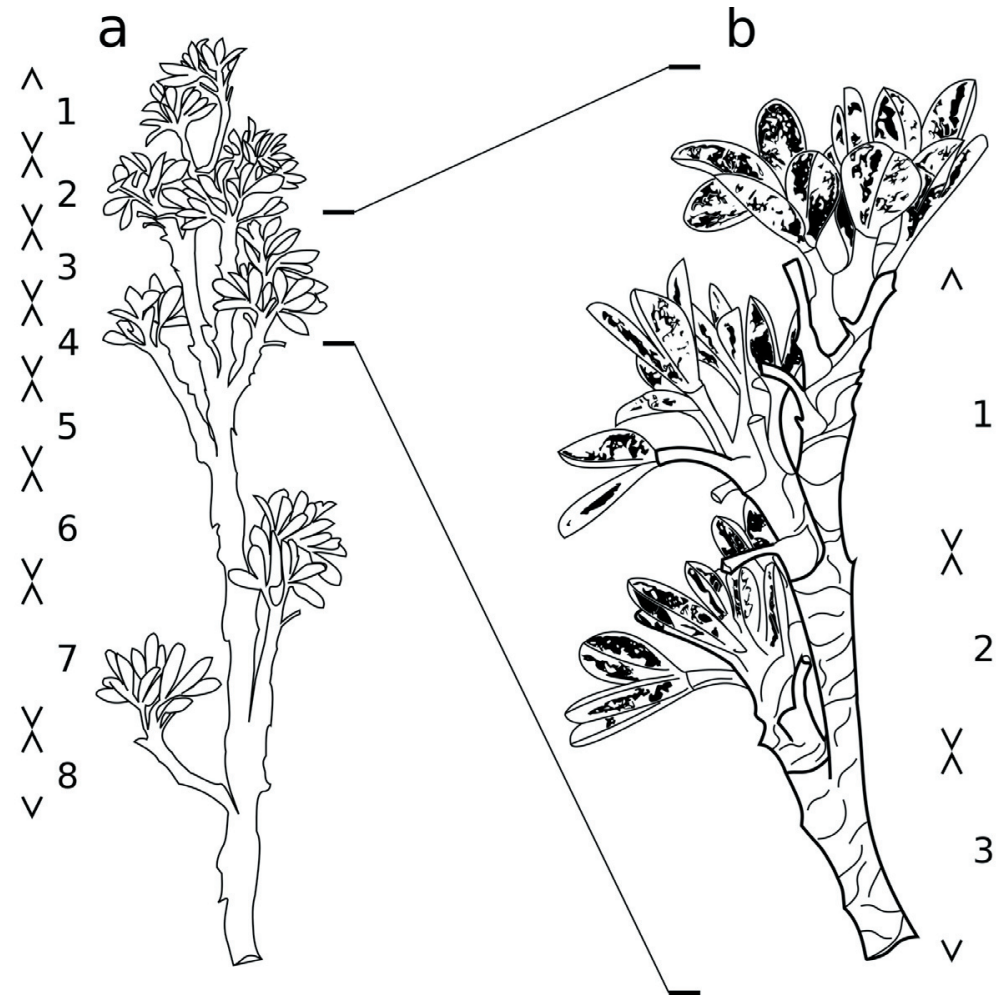

Figura 1. Rama de P. tarapacana. (a) Conteo de marcas de yemas a lo largo de $20 \mathrm{~cm}$ de longitud en la rama principal; (b) brote lateral de la rama principal en donde se contaron 3 marcas. Los números y flechas representan los segmentos entre las marcas de yemas.

Figure 1. Branch of $P$. tarapacana. (a) Count of shoots marks along $20 \mathrm{~cm}$ in length on the main

branch; (b) lateral shoot of the main branch where 3 marks were counted. The numbers and arrows represent the segments between the shoots marks. 
$20 \mathrm{~cm}$ de longitud de la rama principal y b) en un brote lateral de la rama principal se contaron 3 marcas a lo largo del brote (Figura 1). Se determinó la edad a través del conteo de anillos de crecimiento en la base de cada rama de $20 \mathrm{~cm}$ (a) y en la base de cada brote lateral (b). Para ello se realizó el pulido con lijas de diferente textura de progresión ascendente (desde grano 60 a 600) hasta mostrar un brillo espejado sin ralladuras para visualizar con lupa los anillos de crecimiento.

\section{Análisis de datos}

Las características topográficas (altitud, pendiente y orientación) y de estructura forestal (altura dominante del bosque y diámetro del árbol de mayor diámetro) del conjunto de los sitios de estudio se describieron mediante los promedios, los valores mínimos y los valores máximos.

Se estimó el número de marcas de crecimiento medio y sus intervalos de confianza (IC) para ramas de 3 marcas y ramas de $20 \mathrm{~cm}$. Se realizó un análisis de Glmm (Generalized Linear Mixed-Effects Model) mediante la utilización del paquete Lme4 (Bates et al. 2015) en el software R (R Core Team 2020), utilizando el conteo de marcas de yemas a lo largo de la rama como variable explicativa y la edad determinada por conteo de anillos de crecimiento como variable de respuesta. Se incluyó en el modelo el factor aleatorio Árbol ( $n=26$, obtenidas de 13 árboles). Se calcularon los intervalos de confianza de los parámetros de la regresión (ordenada al origen y pendiente) y, además, se incluyó en el gráfico de regresión los intervalos al 95\% para la recta (Figura 2). Los intervalos de confianza de los parámetros de la regresión se calcularon con el fin de observar si dentro de los IC se encuentra el valor 0 de ordenada al origen y la pendiente 1 , indicadores de

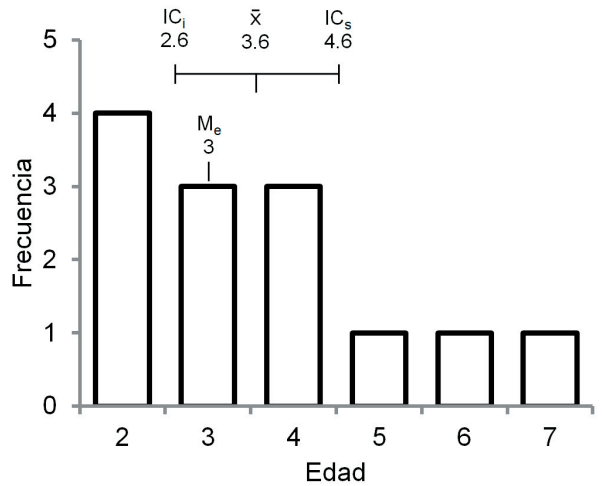

Figura 2. Histograma de frecuencias entre las edades medidas de todas las ramas de tres marcas, incluyendo la edad media, los intervalos de confianza y la mediana. $\mathrm{M}_{\mathrm{e}}$ : Mediana; $\bar{x}$ : Media aritmética; $\mathrm{IC}_{\mathrm{i}}$ : Intervalo de confianza inferior; $\mathrm{IC}_{\mathrm{S}}$ : Intervalo de confianza superior.

Figure 2. Frequency histogram between the measured ages of all the branches of three shoot marks, including the mean age, confidence intervals and median. $\mathrm{M}$ : Median; $\overline{\mathrm{x}}$ :Arithmetic mean; $\mathrm{IC}_{\mathrm{i}}$ : Lower confidence interval; $\mathrm{IC}_{\mathrm{s}}$ : Upper confidence interval.

una relación 1:1 entre las marcas y la edad. Se confeccionó un histograma de frecuencias entre las edades medidas de todas las ramas de 3 marcas incluyendo la edad media, los intervalos de confianza al $95 \%$ y la mediana como información en el gráfico.

\section{Resultados}

Los 13 sitios estudiados presentan una amplia heterogeneidad en variables topográficas (i.e., altitud, exposición y pendiente) donde la amplitud del gradiente altitudinal fue de 537 $\mathrm{m}$, las exposiciones en donde se desarrollan estos bosques abarcan los 4 puntos cardinales, y las pendientes fueron moderadas a altas, sin observar bosques en pendientes menores a $17^{\circ}$. A su vez, los bosques presentan estructuras forestales contrastantes en altura dominante y DAP máximo (Tabla 1).

Tabla 1. Valores promedio, máximos y mínimos de las variables topográficas y de estructura forestal de los 13 bosques de P. tarapacana estudiados. Altitud: Altitud en m s. n. m.; ExE: Exposición este (seno de la exposición en grados [oscila entre - 1 en el oeste y 1 en el este]); ExN: Exposición norte (coseno de la exposición en grados [oscila entre -1 en el sur y 1 en el norte]); Pend: Pendiente en grados; HtDom: Altura dominante del bosque como promedio de los tres árboles más altos; DAPmax: Diámetro $(\mathrm{cm})$ a $1.3 \mathrm{~m}$ del árbol de mayor diámetro; Prom: Promedio; Mín: Mínimo; Máx: Máximo,

Table 1. Average, maximum and minimum of surveyed variables and forest structure of 13 P. tarapacana studied forests. Altitud: Altitude in m a. s. 1.; ExE: East exposure (sine of exposure in degrees [ranging from -1 in the west to 1 in the east]); ExN: North exposure (cosine of exposure in degrees [ranging from -1 in the south to 1 in the north]); Pend: Slope in degrees; HtDom: dominant height of the forest as average of the three tallest trees; DAPmax: Diameter $(\mathrm{cm})$ at 1.3 $\mathrm{m}$ of the tree with the largest diameter; Prom: Average; Mín: Minimum; Máx: Maximum.

\begin{tabular}{lcccccc}
\hline & $\begin{array}{c}\text { Altitud } \\
(\mathrm{m} \mathrm{s.n.} \text { m. })\end{array}$ & $\begin{array}{c}\text { ExE } \\
(\text { seno })\end{array}$ & $\begin{array}{c}\text { ExN } \\
(\text { coseno })\end{array}$ & $\begin{array}{c}\text { Pend } \\
\left({ }^{\circ}\right)\end{array}$ & $\begin{array}{c}\text { HtDom } \\
(\mathrm{cm})\end{array}$ & $\begin{array}{c}\text { DAPmax } \\
(\mathrm{cm})\end{array}$ \\
\hline Prom & 4559 & 0.30 & 0.66 & 23.3 & 157.7 & 3.6 \\
Mín & 4305 & -0.57 & -0.95 & 17.0 & 81.6 & 0.0 \\
Máx & 4842 & 0.98 & 0.99 & 33.0 & 241.6 & 12.0 \\
\hline
\end{tabular}




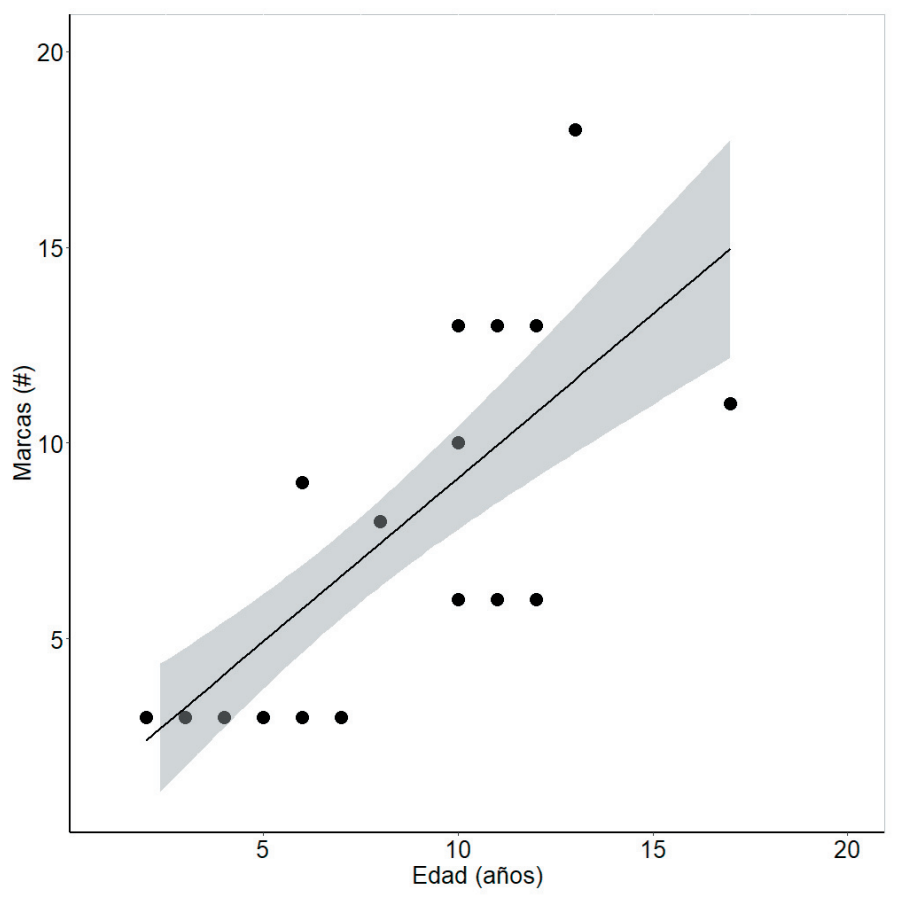

Figura 3. Relación entre el número de marcas a lo largo de la rama y la edad medida por recuento de anillos de crecimiento en ramas de $P$. tarapacana. El sombreado representa el intervalo de confianza del modelo al 95\%.

Figure 3. Relationship between number of shoot marks along the branch and the age measured by counting growth rings in branches of $P$. tarapacana. The shaded area represents the $95 \%$ confidence interval of the model.
Los intervalos de confianza presentaron valores de 0.9 años para las ramas con 3 marcas y de 3.2 años para las de $20 \mathrm{~cm}$ de longitud. El histograma de frecuencias entre las edades medidas de todas las ramas de 3 marcas presentó una edad media de 3.6 años, valor superior a los 3 años de edad. Sin embargo, la relación 1:1 (Marcas y Edad) se encuentra dentro del intervalo de confianza calculado. La mediana presentó un valor de 3 años. El análisis Glmm presentó los siguientes valores para la ordenada al origen $=2.11904 ; P=0.0347$; IC $=0.26353$ a 3.97442 y para el parámetro de la pendiente del modelo $=0.74665 ; P<0.0001$; IC $=0.51521$ a 0.97796 . Las marcas influyeron significativamente en la edad de las ramas $(\mathrm{p}<0.0001)$ y no hubo efecto del factor árbol. En la Figura 3 se observa la relación entre el número de marcas a lo largo de la rama y la edad medida por recuento de anillos de crecimiento y se presentan los intervalos de confianza al $95 \%$ para la recta.

\section{Discusión}

La técnica de predicción de la edad en brotes mediante marcas externas de yemas a lo largo de la rama se basa en que los brotes anuales del tallo principal pasan por la fase de yema antes dedesarrollarse (Kozlowski and Pallardy 1997). En ramas jóvenes, estos brotes anuales podrían detectarse retrospectivamente mediante estas marcas, que indican la ubicación en donde termina un año de crecimiento y comienza el siguiente (Williams and Johnson 1990). Los recuentos de marcas de yemas sólo son viables como registros de la edad del árbol cuando las marcas son visibles, ya que a medida que el tallo se expande radialmente las marcas desaparecen de manera progresiva (Hett and Loucks 1976; Thabeet et al. 2009). La estimación de la edad mediante esta técnica es una herramienta rápida, precisa y fácil de implementar a campo (siempre y cuando estas marcas sean visibles) sin tener que colectar muestras y analizarlas luego en laboratorio. Existe un límite de edad en el que éstas no se aprecian con claridad (Hett and Loucks 1976; Williams and Johnson 1990; Thabeet et al. 2009).

Las formaciones forestales de $P$. tarapacana se desarrollan en un rango altitudinal de 40005000 m s. n. m. (Kessler 2006; Renison et al. 2013), en coincidencia con el rango observado en la toma de muestras del presente trabajo (4304.8 a 4841.9 m s. n. m.). Las pendientes analizadas no contemplan situaciones menores a $17^{\circ}$, un resultado similar al que observaron Saavedra (2013) y López et al. (2020), quienes encontraron que las pendientes donde se desarrollan estos bosques presentan valores entre 15 a $20^{\circ}$. Sin embargo, Ríos (1998) describe bosques en menores pendientes en Tarapacá, Chile. Las variables de estructura forestal presentaron una variación acorde a otros estudios de estructura de bosques de P. tarapacana (Ríos 1998; Saavedra 2013; 
López et al. 2020). Los rangos ambientales se ajustaron a lo descrito por otros autores (Ríos 1998; Saavedra 2013; López et al. 2020), por lo que con esta amplitud ambiental sería posible evaluar la potencialidad de esta técnica (Tabla 1).

Este estudio presentó resultados similares a los observados por Urza and Sibold (2013) y por Hankin et al. (2018) en coníferas; en estos trabajos, el recuento de marcas subestimó la edad de los brotes. En el caso de Polylepis en general, y $P$. tarapacana en particular, existe una evidente producción de corteza (Kessler 2006), lo que resulta en un ocultamiento directamente proporcional a la edad de la marca. Sin embargo, en la estimación de la edad en ramas con 3 marcas, tanto la mediana como el intervalo de confianza incluyen el mismo valor de marcas y edad, lo que le da a esta técnica una potencialidad de uso para esta especie. Es necesario realizar futuras investigaciones para evaluar los resultados para otros intervalos.

En este estudio, la ordenada al origen del Glmm (Generalized Linear Mixed-Effects Model), presentó un valor positivo y el IC no incluyó como valor al 0 . De este modo, para ramas/brotes con una sola marca se estima una edad aproximada de 3 años. Esto podría ocurrir debido a que el límite inferior del modelo lo componen datos de 3 marcas, lo que implica un sesgo causado por el desbalance de los datos o un error en las estimaciones debido al bajo número de muestras. Asimismo, podrían existir un desfasaje entre el número de marcas y la edad medida debido al nulo crecimiento en altura en años desfavorables.

La pendiente de la relación entre el número de marcas y la edad presento un valor inferior a 1 y sin incluir este valor dentro del intervalo de confianza, al igual que en otros estudios que se realizaron en coníferas (Urza and Sibold 2013; Hankin et al. 2018). Una de las limitaciones de este estudio es el bajo tamaño de muestra ( $n=26$, obtenidas de 13 árboles), ya que se colectó una sola rama por cada bosque y es posible que no refleje la variabilidad dentro de un individuo y del bosque. Sin embargo, esta técnica podría verificarse con un mayor tamaño muestral y con mediciones de edad en todas las marcas a lo largo de la rama. Los resultados de este trabajo indican la potencialidad de la utilización de esta técnica. Con la validación de esta técnica para $P$. tarapacana se podría analizar la respuesta en el crecimiento en altura en relación con diversas variables ambientales, como la precipitación en un período de tiempo, midiendo la longitud en brotes del período y de cada año para realizar comparaciones.

Esta metodología de recuento de marcas de yemas en ramas se utilizó en trabajos sobre $N$. pumilio; en los mismos, la técnica fue validada por Cuevas (2002), y luego Gea et al. (2004) y Soler et al. (2018) la aplicaron en estudios de crecimiento. A su vez, esta técnica, validada para renovales de P. pumila (Kajimoto et al. 1998), fue aplicada en diferentes especies de la familia Pinaceae, incluyendo Abies alba Mill., Pinus halepensis Mill., Pinus nigra J. F. Arnold, Pinus pinea L., Pinus pinaster Aiton y Pinus sylvestris L. (Mutke et al. 2005; Thabeet et al. 2009; Crone et al. 2011; Girard et al. 2011; Vennetier et al. 2013). En el caso de Picea engelmannii Parry ex Engelm, $P$. menziesii, Pinus contorta Douglas y Larix occidentalis Nutt, la precisión del método varía con la especie estudiada y la edad estimada (Urza and Sibold 2013). Se observaron errores promedio entre 0.18 y 1.25 años y los desvíos estándar variaron entre 1.83 a 2.70 años para estimaciones de 2 a 21 años. Estos autores reportaron que el conteo de marcas a lo largo de la rama subestimó la edad en un promedio de 4.1 años, con un sesgo que aumentó con la edad de la marca. Al observarse este sesgo creciente, es fundamental seleccionar los métodos para determinar la edad en función de la precisión necesaria para responder a preguntas ecológicas específicas (Hankin et al. 2018) y remarca la importancia de validar este tipo de técnicas.

Asimismo, las observaciones de campo mostraron que la identificación de marcas de yemas terminales para $P$. engelmannii, $P$. menziesii, $P$. contorta y $L$. occidentalis se encuentra más limitada por incrementos de crecimiento extremadamente estrechos que por marcas ocultas por la formación de la corteza (Urza and Sibold 2013). En el caso de $P$. menziesii, el sesgo aumentó con la edad de la muestra más rápido en los individuos de crecimiento más lento, lo que sugiere que estos individuos podrían producir nodos anuales poco claros debido a limitaciones fisiológicas (Hankin et al. 2018). En el caso de P. tarapacana, esta especie presenta un lento crecimiento, lo que puede dificultar la determinación de marcas en años particularmente secos (Hoch and Körner 2005).

Hoch and Körner (2005) utilizaron esta metodología en $P$. tarapacana y calcularon el incremento anual de las ramas apicales 
utilizando la longitud de dos años de crecimiento en el brote terminal sin realizar una corroboración de la precisión de la técnica. Aplicar el método de conteo de marcas no daña a las plantas, es rápido y se puede realizar en el campo sin equipo especializado. Las marcas de yemas de los últimos años se pueden identificar y se pueden obtener tasas de crecimiento vertical de los últimos años sin mediciones directas en cada año.

En el presente trabajo se evaluó la potencialidad de la técnica de estimación de la edad, que utiliza las marcas externas a lo largo de la rama en $P$. tarapacana como caso de estudio, obteniendo resultados alentadores. Sin embargo, para aplicar esta técnica es fundamental conocer cuándo las marcas de las yemas producen una estimación precisa de la edad de los brotes. Si bien nuestros resultados muestran la potencialidad del uso de marcas para estimar la edad (siendo una técnica no destructiva) los niveles de precisión y error son altos debido al bajo número de muestras analizadas. Tanto en este estudio como en otras especies se observó una subestimación de la edad al utilizar el conteo de marcas. Serán necesarias futuras investigaciones para validar si existe una relación 1:1, o para construir un modelo empírico con un muestreo más amplio y mejor balanceado, que además sea validado con datos externos.

Agradecimientos. Deseamos expresar nuestro agradecimiento a la Dra. Mónica Adriana Murace por la facilitación del instrumental para la medición de las muestras y a la Lic. Ainhoa Valbuena por la ayuda durante los muestreos a campo, como así también a la comunidad de Lagunillas del Farallón por el apoyo y ayuda que nos brindaron durante los días de campaña. A su vez, queremos agradecer a Adrián Jáuregui por su ayuda y guía en el análisis estadístico y a las valiosas sugerencias realizadas por los revisores anónimos de este trabajo.

\section{REFERENCIAS}

Assmann, E. 1970. The principles of forest yield study: Studies in the organic production, structure, increment, and yield of forest stands. Pergamon Press, Oxford, England.

Bates, D., M. Maechler, B. Bolker, and S. Walker. 2015. Fitting Linear Mixed-Effects Models Using lme4. Journal of Statistical Software 67(1):1-48. https://doi.org/10.18637/jss.v067.i01.

Braun, G. 1997. The use of digital methods in assessing forest patterns in an Andean environment: the Polylepis example. Mountain Research and Development 17:253-262. https://doi.org/10.2307/3673852.

Cozzi, E. A., and F. Moschione. 2011. Guía de flora y fauna en la región de Mina Pirquitas. Mina Pirquitas inc. Jujuy. Argentina.

Crone, E. E., E. J. B. McIntire, and J. Brodie. 2011. What defines mast seeding? Spatio-temporal patterns of cone production by whitebark pine. Journal of Ecology 99:438-444. https://doi.org/10.1111/j.1365-2745.2010.01790.x.

Cuevas, J. G. 2002. Episodic regeneration at the Nothofagus pumilio alpine timberline in Tierra del Fuego, Chile. Journal of Ecology 90:52-60. https://doi.org/10.1046/j.0022-0477.2001.00636.x.

Cuyckens, G. A. E., D. A. Christie, A. I. Domic, L. R. Malizia, and D. Renison. 2016. Climate change and the distribution and conservation of the world's highest elevation woodlands in the South American Altiplano. Global and Planetary Change 137:79-87. https://doi.org/10.1016/j.gloplacha.2015.12.010.

Gea-Izquierdo, G., G. Martínez Pastur, J. M. Cellini, and M. V. Lencinas. 2004. Forty years of silvicultural management in southern Nothofagus pumilio primary forests. Forest Ecology and Management 201:335-347. https://doi.org/10.1016/ j.foreco.2004.07.015.

Girard, F., M. Vennetier, F. Guibal, C. Corona, S. Ouarmim, and A. Herrero. 2011. Pinus halepensis Mill. crown development and fruiting declined with repeated drought in Mediterranean France. European Journal of Forest Research 131:919-931. https://doi.org/10.1007/s10342-011-0565-6.

Hallé, F., R. A. A. Oldeman, and P. B. Tomlinson. 1978. Tropical Trees and Forests. An Architectural Analysis. SpringerVerlag, Berlin, Germany. https://doi.org/10.1007/978-3-642-81190-6.

Hankin L. E., P. E. Higuera, K. T. Davis, and S. Z. Dobrowski. 2018. Accuracy of node and bud-scar counts for aging two dominant conifers in western North America. Forest Ecology and Management 427:365-371. https://doi.org/ 10.1016/j.foreco.2018.06.001.

Hett, J. M., and O. L. Loucks. 1976. Age structure models of balsam fir and eastern hemlock. Journal of Ecology 64: 1029-1044. https://doi.org/10.2307/2258822.

Hoch, G., and C. Körner. 2005. Growth, demography and carbon relations of Polylepis trees at the world's highest treeline. Functional Ecology 19:941-951. https://doi.org/10.1111/j.1365-2435.2005.01040.x.

IUCN. Red List of Threatened Species. Version 2020. URL: https://www.iucnredlist.org.

Kajimoto, T., H. Onodera, S. Ikeda, H. Daimaru, and T. Seki. 1998. Seedling establishment of subalpine stone pine (Pinus pumila) by nutcracker (Nucifraga) seed dispersal on Mt. Yumori, Northern Japan. Arctic and Alpine Research 30:408-417. https://doi.org/10.2307/1552014.

Kessler, M. 1995. Present and potential distribution of Polylepis (Rosaceae) forests in Bolivia. Pp. 281-294 in Proceedings 
of the neotropical montane forest biodiversity and conservation symposium. The New York Botanical Garden. New York. USA.

Kessler, M. 2006. Bosques de Polylepis. Pp. 110-120 in M. Moraes, B. Øllgaard, L. P. Kvist, F. Borchsenius and H. Balslev (eds.). Botánica Económica de los Andes Centrales. Universidad Mayor de San Andrés, La Paz, Bolivia.

Kozlowski, T. T., and S. G. Pallardy. 1997. Physiology of woody plants. Second edition. Academic, California, USA.

López, V. L., M. Pérez Flores, S. E. Sharry, and J. M. Cellini. 2020. Estructura vertical y horizontal en dos bosques de Polylepis tarapacana con diferentes situaciones ambientales. Historia Natural 10:165-173.

Martínez Pastur, G., M. V. Lencinas, P. Peri, and M. Arena. 2007. Photosynthetic plasticity of Nothofagus pumilio seedlings to light intensity and soil moisture. Forest Ecology and Management 243:274-282. https://doi.org/10.1016/ j.foreco.2007.03.034.

Mendoza, E. A., and J. A. González. 2011. Las ecorregiones del Noroeste Argentino basadas en la clasificación climática de Köppen. Serie Conservación de la Naturaleza 19:3-41.

Miehe, G., S. Miehe, K. Koch, and M. Will. 2003. Sacred forests in Tibet using geographical information systems for forest rehabilitation. Mountain Research and Development 23:324-328. https://doi.org/10.1659/0276-4741(2003)023[0324: SFIT]2.0.CO;2.

Mina Pirquitas. 2004. Registro de datos meteorológicos del área de influencia de la Mina Pirquitas Inc. SSR Mining, Jujuy, Argentina. Pp. 51.

Morales, M. S., R. Villalba, H. R. Grau, and L. Paolini. 2004. Rainfall controlled tree growth in high elevation subtropical treelines. Ecological Society of America 85:3080-3089. https://doi.org/10.1890/04-0139.

Morales, M. S., D. A. Christie, R. Villalba, J. Argollo, J. Pacajes, J. S. Silva, C. A. Álvarez, J. C. Llancabure, and C. C. Soliz Gamboa. 2012. Precipitation changes in the South American Altiplano since 1300 AD reconstructed by tree-rings. Climate of the Past 8:653-666. https://doi.org/10.5194/cp-8-653-2012.

Mutke, S., R. Sievänen, E. Nikinmaa, J. Perttunen, and L. Gil. 2005. Crown architecture of grafted Stone pine (Pinus pinea L.): shoot growth and bud differentiation. Trees 19:15-25.

Piña, P. Z. 2015. Caracterización de hábitat y distribución espacial de formaciones boscosas de queñoa (Polylepis tarapacana) en los territorios del Parque Nacional Salar del Huasco, región de Tarapacá. Biodiversidata 3:12-20.

R Core Team. 2020. R: A language and environment for statistical computing. R Foundation for Statistical Computing, Vienna, Austria. URL: https://www.R-project.org.

Renison, D., I. Hensen, R. Suárez, A. M. Cingolani, P. Marcora, and M. A. Giorgis. 2010. Soil conservation in Polylepis mountain forests of Central Argentina: Is livestock reducing our natural capital? Austral Ecology 35:435-443. https: //doi.org/10.1111/j.1442-9993.2009.02055.x.

Renison, D., G. A. E. Cuyckens, S. Pacheco, G. F. Guzmán, H. R. Grau, P. Marcora, G. Robledo, A. M. Cingolani, J. Dominguez, M. Landi, L. Bellis, and I. Hensen. 2013. Distribución y estado de conservación de las poblaciones de árboles y arbustos del género Polylepis (Rosaceae) en las montañas de Argentina. Ecología Austral 23:27-36. https: //doi.org/10.25260/EA.13.23.1.0.1189.

Ríos, S. 1998. Estudio de la distribución y caracterización ecológica de las poblaciones locales de queñoa de altura (Polylepis tarapacana Phil.) en el sector de la provincia de Iquique. Tarapacá. Chile. Memoria Ing. Forestal. Facultad de Ciencias Agrarias y Forestales, Universidad de Chile. Santiago de Chile. Chile. Pp. 61

Saavedra, J. 2013. Caracterización de la estructura poblacional de Polylepis tarapacana en sector cerro Chiguana, cuenca del salar de Surire, para establecer los lineamientos de un programa de restauración ecológica. Tesis de Maestría. Universidad de Tarapacá. Arica. Chile. Pp. 76.

Soler, R., M. V. Lencinas, G. Bustamante, and G. Martínez Pastur. 2018. Atributos de la regeneración natural de ñire (Nothofagus antarctica) en Tierra del Fuego: beneficios y perjuicios que genera el uso silvopastoril. Ecosistemas 27(3): 41-47.

Thabeet, A., M. Vennetier, C. Gadbin-Henry, N. Denelle, M. Roux, Y. Caraglio, and B. Vilá. 2009. Response of Pinus sylvestris L. to recent climatic events in the French Mediterranean region. Trees 23:843-853. https://doi.org/10.1007/ s00468-009-0326-z.

Toivonen, J. M., V. Horna, M. Kessler, K. Ruokolainen, and D. Hertel. 2014. Interspecific variation in functional traits in relation to species climatic niche optima in Andean Polylepis (Rosaceae) tree species: evidence for climatic adaptations. Functional Plant Biology 41:301-312. https://doi.org/10.1071/FP13210.

Urza, A. K., and J. S. Sibold. 2013. Nondestructive Aging of Postfire Seedlings for Four Conifer Species in Northwestern Montana. Western Journal of Applied Forestry 28(1):22-29. https://doi.org/10.5849/wjaf.11-014.

Vennetier, M., F. Girard, O. Taugourdeau, M. Cailleret, Y. Caraglio, S. A. Sabatier, S. Ouarmim, D. Cody, and A. Thabeet. 2013. Climate change impact on tree architectural development and leaf area. Pp. 103-126 in Singh Bharat Raj (ed.). Climate change - realities, impacts over ice cap, sea level and risks. InTech. Rijeka, Croacia. https://doi.org/ $10.5772 / 51510$.

Wawrzyk, A. C., and B. L. Vilá. 2013. Dinámica del pastoreo en dos comunidades (Lagunillas del Farallón y Suripujio) de la puna de Jujuy, Argentina. Chungara: Revista de Antropología Chilena 45(2):349-362. https://doi.org/10.4067/ S0717-73562013000200010.

Williams, C. E., and W. C. Johnson. 1990. Age structure and the maintenance of Pinus pungens in pine-oak forests of southwestern Virginia. American Midland Naturalist 124:130-141. https://doi.org/10.2307/2426086.

Zhang, L., T. Luo, X. Liu, and G. Kong. 2010. Altitudinal variations in seedling and sapling density and age structure of timberline tree species in the Sergyemla Mountains, southeast Tibet. Acta Ecologica Sinica 30(2):76-80. https: //doi.org/10.1016/j.chnaes.2010.03.005. 